\title{
Special issue on 6th European Conference on Precision Agriculture and 2nd Precision Crop Protection Conference
}

\author{
John V. Stafford
}

This special issue of the Journal concludes publication of enhanced papers from the 6th European Conference on Precision Agriculture (3 papers) and the 2nd Precision Crop Protection Conference (5 papers) both held in 2007. Some 22 good papers from the former and 10 from the latter have now been published bringing together an important body of research from several disciplines to the attention of the precision agriculture research community.

Technology development was a prime driver for precision agriculture in its early phases. With the maturing of the concept, other disciplines (soil science, crop science and many others) have taken their place alongside technology. Technology continues to develop and the developments are being applied to PA as illustrated in the three 6ECPA papers; they describe RFID tags for produce tracing, laser rangefinding applied to field crops and wireless soil sensors.

Precision crop protection includes the investigation of weed, insect and fungal pests, the latter two being the topic of 3 of the 2PCPC papers. Technology features in the last two papers, both dealing with precision spray application. 